\title{
AUTOMATIC BUILDING EXTRACTION FROM LIDAR POINT CLOUD DATA IN THE FUSION OF ORTHOIMAGE
}

\author{
B. Hujebri ${ }^{1,}{ }^{*}$ M. Ebrahimikia ${ }^{2}$, H. Enayati ${ }^{3}$ \\ ${ }^{1} \mathrm{PhD}$ student in Photogrammetry, School of Surveying and Geospatial Engineering, College of Engineering, University of Tehran, \\ North Kargar Ave., Jalal Al. Ahmad Crossing, Tehran, Iran- b.hujebry@ut.ac.ir \\ ${ }^{2}$ PhD student in Remote Sensing, K.N.Toosi University of Technology, Mirdamad, Tehran, Iran - m.ebrahimikia@kntu.ac.ir \\ ${ }^{3}$ MSc degree of Photogrammetry, K.N.Toosi University of Technology, Mirdamad, Tehran, Iran - enayati_hamid@yahoo.com
}

KEY WORDS: Building, Segmentation, Mean Shift, Image, LiDAR, Point Cloud

\begin{abstract}
:
Three-dimensional building models are important in various applications such as disaster management and urban planning. In this paper, a method based on the fusion of LiDAR point cloud and aerial image data sources has been proposed. The first step of the proposed method is to separate ground and non-ground (that contain $3 \mathrm{~d}$ objects like buildings, trees, ...) points using cloth simulation filtering and then normalize the non-ground points. This research experiment applied a 0.1 threshold for the $\mathrm{z}$ component of the normal vector to remove wall points, and 2-meter height threshold to remove off-terrain objects lower than the minimum building height. It is possible to discriminate vegetation and building based on spectral information from orthoimage. After elimination of vegetation points, the mean shift algorithm applied on remaining points to detect buildings. This method provides good performance in dense urban areas with complex ground covering such as trees, shrubs, short walls, and vehicles.
\end{abstract}

\section{INTRODUCTION}

The automatic extraction of accurate building boundaries is important geospatial information that is indispensable for several applications (Gilani, Awrangjeb, \& Lu, 2015). Building information is extremely important for many applications such as urban planning, telecommunication, or environmental monitoring, etc. Automated techniques and tools for data acquisition from remotely sensed imagery are urgently needed. This paper presents an automatic approach for building extraction from airborne LIDAR data.

Researches performed according to different performance criteria such as: data sources, level of user interaction, geometrical accuracy, model integrity, and applications. Although modeling systems differ according to these criteria, obtaining data seriously affects the characteristics and utility of the models. With the development of laser scanner technology, the system has become a viable and conventional data source for detailed mapping of structures. With the evolution of technology related to light detection and distance detection, density and accuracy of points cloud have been steadily increasing. In recent years, several methods have been proposed for building extraction. The algorithms comprise methods that only employ LIDAR point cloud for model generation (M. Awrangjeb, Fraser, \& Lu, 2015; Feng, Zhang, Li, Jin, \& Xia, 2019; R. Huang et al., 2018; Tomljenovic, Tiede, \& Blaschke, 2016; Varghese, Shajahan, \& Nath, 2017) while some others use additional data sources such as aerial or satellite imagery (Akbulut, Özdemir, Acar, \& Karsli, 2018; Mohammad Awrangjeb, Zhang, \& Fraser, 2013; Dal Poz \& Fernandes, 2017; Dong, Kyoung, \& Sang, 2008; J. Huang, Zhang, Xin, Sun, \& Zhang, 2019; Maltezos \& Ioannidis, 2015; Moussa, 2012; Teo \& Chen, 2004; Zhou, Member, \& Zhou, 2014). Therefore, different methods can be categorized into sources based on photogrammetric sensors, active sensors, and combinations of them (Hu, You, \& Neumann, 2000).

The most challenging factor confronted in boundary delineation is building shape variability and surrounding environment complexity. In order to deal with various building types, a new method for automatic building detection through effective integration of LiDAR data and multispectral imagery (Gilani et al., 2015). A data-driven approach was proposed, yields the complementary advantages from both the LiDAR data and multispectral image. The initial building positions are obtained after connected component analysis carried on LiDAR data. Then, the multispectral image is used to eliminate false objects and vegetation, detect buildings, and delineate the corresponding building boundaries. The final building boundary is obtained by extending the initial position using both the data sources. The extracted line features are essential elements to form building outlines and can be useful to reconstruct 3D building models(Tseng \& Hung, 2016). Although most building boundary lines were extracted, building outlines seem to incomplete due to some missing lines. The use of first and intermediate laser returns can significantly improve the detection of building outlines. However, misdetection and double edges cannot be avoided due to some complicated roof structures. Insufficient point density of the could be one of the reasons of getting bad results. An automatic method for the extraction of building roof boundaries with the integration of LiDAR data and the aerial image was presented (Awad, 2017). Polylines extracted from LiDAR data that represent aboveground objects are projected onto the image to extract the same objects in image space. The process of identifying polylines that represent building roof boundaries is performed through the GA algorithm of the MRF-based energy function. Some types of segmentation failures of the LiDAR data caused the incorrect segmentation of the selected sub-image.

A new top-down strategy method is proposed to extract buildings solely based on airborne LiDAR point clouds (R. Huang et al., 2018). They separate the ground and non-ground. Then, a top-level processing is used to recognize building regions via surface characteristics and penetrating capacities, which are calculated based on the object entity replacing the point and segment entities. Finally, non-building points are removed from building regions by a down-level processing.

\footnotetext{
Corresponding author.
} 
However, a few buildings may be erroneously removed when the point density is too low or the size is too small. Also, an active contour algorithm is used based on the fusion of LiDAR data with imagery (Akbulut et al., 2018). In areas where the roof and its surroundings are spectrally similar, the addition of height information allows the roof and ground to be distinguished by the algorithm. Afterward, shadows and vegetation areas were removed from both LiDAR and image data by calculating band ratios to prevent active contour algorithm from expanding to these areas. Active contour algorithm needs mask locations which is created automatically from the image dataset by using morphological operations and optimum iteration number to extract buildings from integrated data. A novel method, DBCS, was proposed to extract buildings from airborne LiDAR point clouds (X. Huang, Cao, \& Cao, 2019). The algorithm is based on a density-based spatial clustering technique, using the spatial index to improve performance. Since the data acquired by LiDAR systems are usually non uniformly distributed, the average point spacing in along and across scan directions is not equal, neighborhood retrieving within an ellipse or a rectangle where its axes conform to the scanning direction may be preferable. Also, some buildings are connected to each other that cannot be differentiated from LiDAR data only.

This paper focuses on the process of the segmentation of individual buildings from airborne LiDAR point clouds of an urban area which contain buildings, trees, cars shrubs, and other different regions. A density-based clustering method (mean shift) is utilized in the automatic individual building segmentation process. In this research, we have used a datadriven method based on using irregular point cloud dataset. Also, orthoimage is used for the detection of vegetation points. boundary points using the topological relationship between the tree boundary and the initial building boundary.

\section{PROPOSED METHOD}

Figure 1. presents the proposed workflow for the extraction of buildings from the LiDAR point cloud.

\subsection{Filtering}

Separating point clouds into the ground and non-ground measurements is an essential step to the detection of buildings on the terrain surface from LiDAR data. However, most filtering algorithms need to carefully set up a number of complicated parameters to achieve high accuracy.

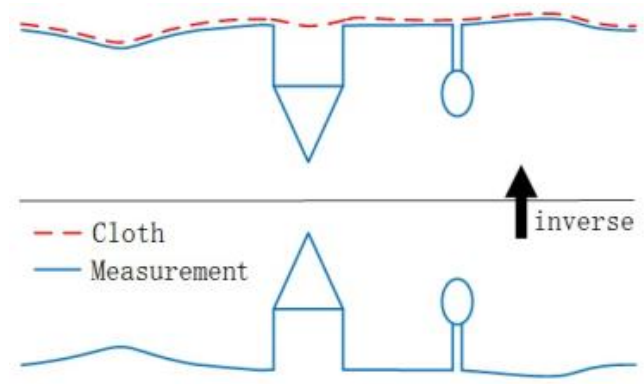

Figure 2. Overview of the cloth simulation algorithm (Zhang et al., 2016)

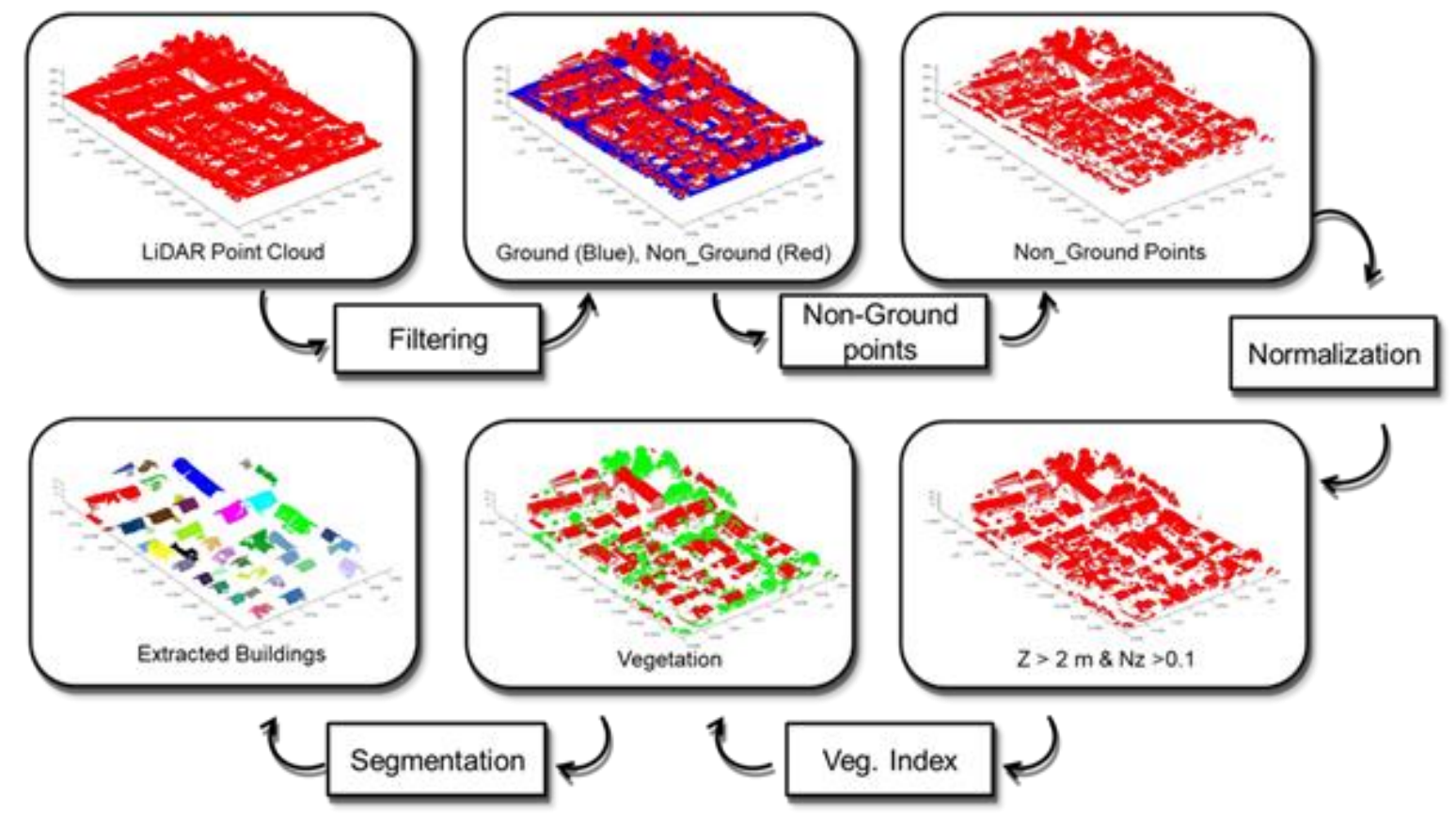

Figure 1. Flowchart of the proposed method

Based on the RMBR algorithm, an IMBR algorithm proposed to extract a complete and accurate regularized boundary of a partially occluded building with orthogonal or parallel sides (Feng et al., 2019). The proposed algorithm avoids the influence of false initial boundary points on the MBR during each step and obtains an accurate regularized boundary. The IMBR algorithm automatically determines the false initial
In this research, we used a filtering method which only needs a few easy-to-set integers and Boolean parameters (Zhang et al., 2016). Within this approach, a LiDAR point cloud is inverted, and then a rigid cloth is used to cover the inverted surface. By analyzing the interactions between the cloth nodes and the corresponding LiDAR points, the locations of the cloth nodes can be determined to generate an approximation of the ground surface. Finally, the ground points can be extracted from the 
LiDAR point cloud by comparing the original LiDAR points and the generated surface. The output of the filtering method is illustrated in figure 3.

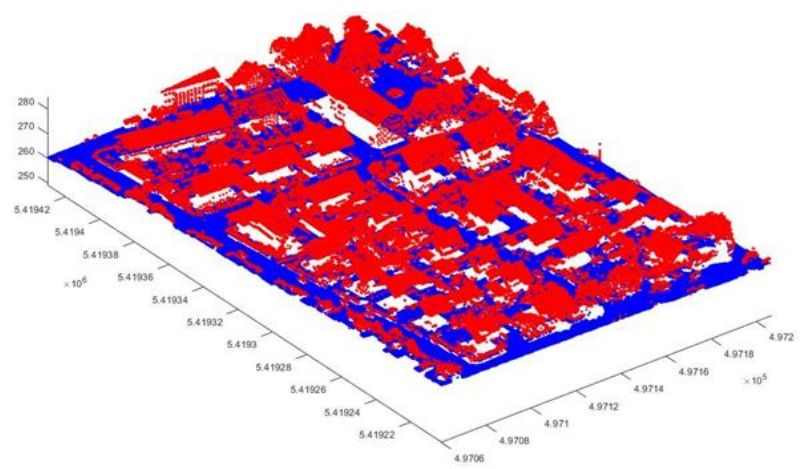

Figure 3. Result of filtering, blue (ground points) and red (above ground points such as: tree, building, car, ...)

\subsection{Elimination of near ground and wall points}

In order to eliminate wall points, the third element of the normal vector. In this paper PCA (Liu \& Mason, 2009) algorithm has been used to calculate the normal vector and the experiment applied a 0.1 threshold on the third component of the normal vector for eliminating wall points, also 2-meter height threshold to remove non-ground objects lower than the minimum building height. Figure 4 shows the output of this stage.

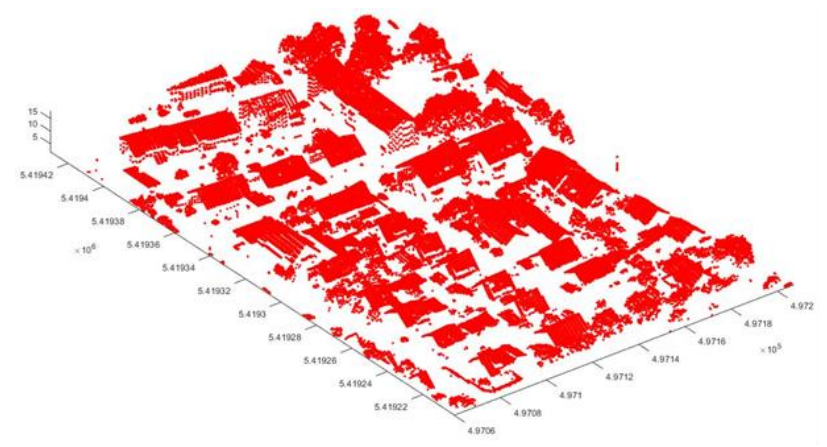

Figure 4. Dataset after elimination of points that belong to vertical objects such as: wall points

\subsection{Vegetation detection}

Additional reflectance information delivered by some airborne laser scanner sensors or multi-spectral imagery may be useful to distinguish vegetation from buildings. In this paper buildings and vegetation are discriminated by utilization of "EXG" vegetation index. In figure 5 vegetations are represented by green points.

$\mathrm{EXG}=2 * \rho_{\text {green }}-\rho_{\text {red }}-\rho_{\text {blue }}$

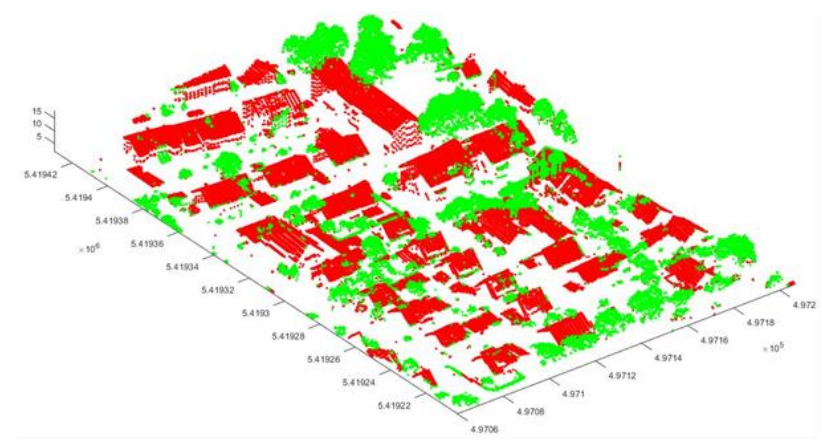

\subsection{Mean Shift Segmentation}

Mean-shift is an algorithm for nonparametric density gradient estimation using a kernel. A mode means a local density maximum. It was first proposed by Fukunaga and Hostetler to calculate density gradient (Fukunaga \& Hostetler, 1975).

The main idea behind the mean shift is to treat the points in the d-dimensional feature space as an empirical probability density function where dense regions in the feature space correspond to the local maxima or modes of the underlying distribution (Derpanis, 2005). For each data point in the feature space, one performs a gradient ascent procedure on the local estimated density until convergence. The stationary points of this procedure represent the modes of the distribution. Furthermore, the data points associated (at least approximately) with the same stationary point are considered members of the same cluster.

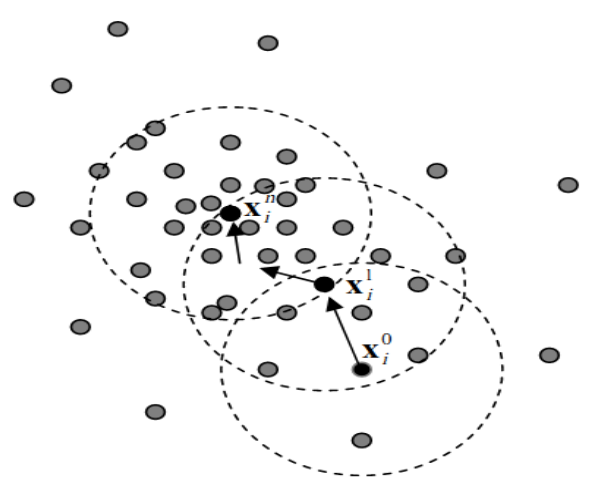

Figure 6. Mean shift procedure (Derpanis, 2005)

\section{EXPERIMENT AND RESULT}

The presented algorithm is applied to the Vaihingen test data set provided by DGPF also contains Airborne Laserscanner (ALS) data. The entire DGPF data set consists of 10 ALS strips acquired on 21 August 2008 by Leica Geosystems using a Leica ALS50 system with $45^{\circ}$ field of view and a mean flying height above ground of $500^{\circ} \mathrm{m}$. The median point density is 6.7 points / m2. Point density varies considerably over the whole block depending on the overlap, but in regions covered by only one strip, the mean point density is 4 points $/ \mathrm{m} 2$. Figure 7 illustrates the LiDAR data of residential areas and orthoimage of the test area is shown in Figure 8. 


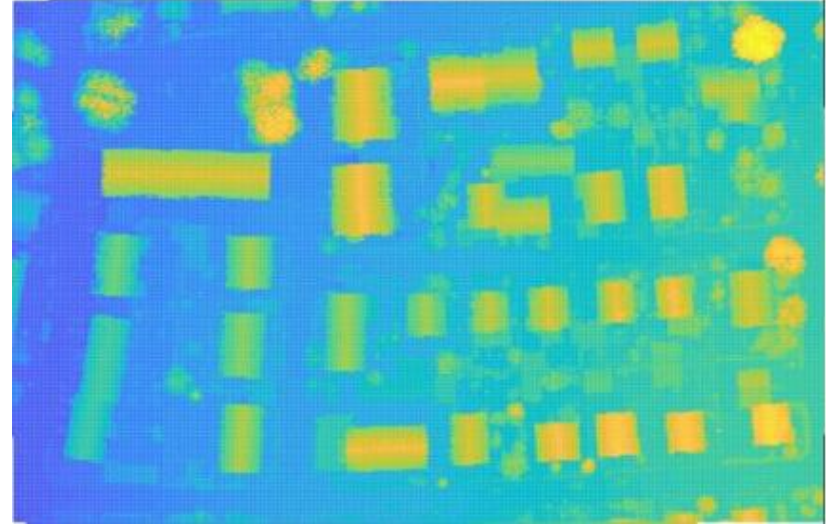

Figure 7. The ALS data of the Vaihingen test site

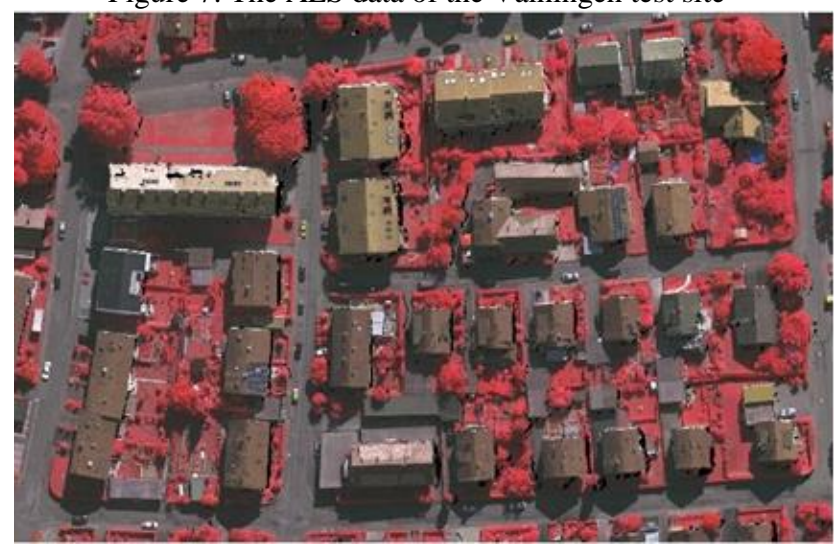

Figure 8. The orthoimage of the test site

Figure 6 illustrates the final result of extracted buildings by the proposed approach. Ground reference data for existing buildings in the study area is also shown in Figure 7. By comparing these two images, the efficiency and capability of the proposed method can be visually evaluated.

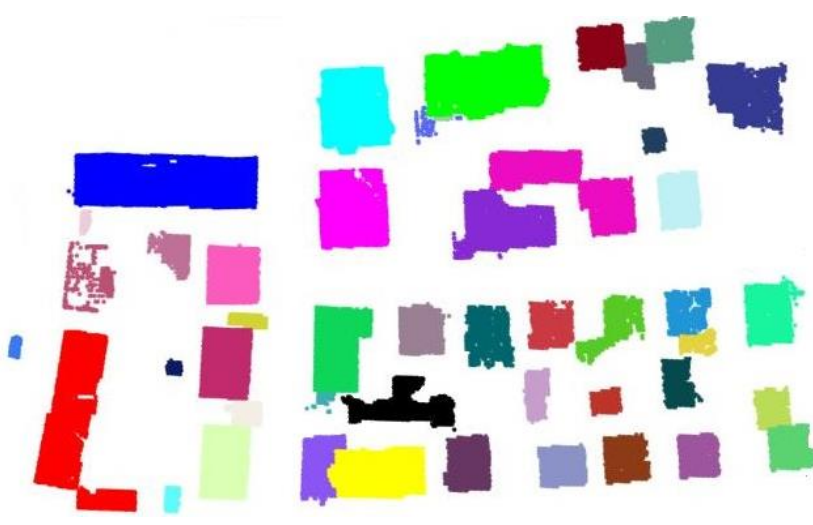

Figure 9. Detected buildings by the proposed method
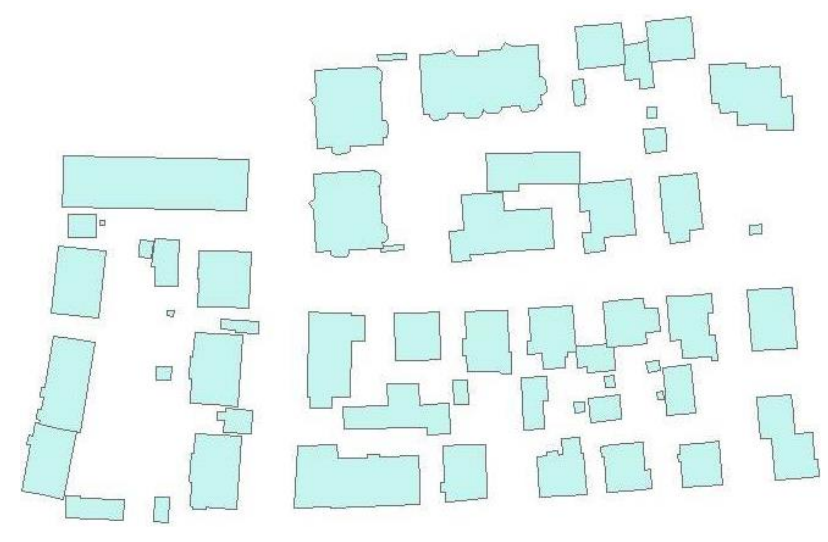

Figure 10. Reference data for building extraction

\subsection{QUANTITATIVE CRITERIA}

For evaluation of the building extraction quality, the results of the automatic procedure and also the reference building database were compared. The extracted buildings were compared one by one to the buildings in reference data (ground truth). The standard statistical parameters are defined and measured as follows (Haala and Brenner, 1999):

1. True positive (TP) - both the automated method and the reference building database label a point as a building;

2. True negative (TN) - both the automated method and the reference building database label a point as background (non-building);

3. False-positive (FP) - only the automated method labels a point as a building;

4. False-negative (FN) -only the reference building database labels a point as a building;

Using these four categories, the following statistical measures were computed to evaluate the performance of the automated building extraction process.

$$
\begin{array}{ll}
\text { Branching Factor }=\frac{F P}{T P} & \text { Detection Ration }=\frac{T P}{T P+F N} \\
\text { Correctness }=\frac{T P}{T P+F P} & \text { Completeness }=\frac{T P}{T P+F N} \\
\text { Miss Factor }=\frac{F N}{T P} & \text { Quality Ration }=\frac{T P}{T P+F P+F N}
\end{array}
$$

Number of buildings in ground reference data: 57

Number of detected buildings: 46

$\mathrm{TP}=45$

$\mathrm{FP}=1$

$\mathrm{FN}=12$

$\begin{array}{ll}\text { Branching Factor }=0.022 & \text { Detection Ration }=0.789 \\ \text { Correctness }=0.978 & \text { Completeness }=0.789 \\ \text { Miss Factor }=0.267 & \text { Quality Ration }=0.776\end{array}$

As shown in Figure 9 and Figure 10 most of the buildings that are not identified by the method are small buildings. This is due to the definition of the altitude threshold and the minimum number of points in each section. 


\section{CONCLUSION}

In this study, using different features of irregular lidar point cloud and orthoimage, a method was proposed for building detection and extraction. First, using the simulation filtering method, the point cloud is divided into two groups of and nonground points, and then the lidar point cloud is converted into normalized points by the difference of the height of the ground points from the non-ground points. Then, using a height threshold, points less than $2 \mathrm{~m}$ in height are excluded from the dataset. Then, using normal vector analysis, the points on the walls and vertical facets are also eliminated. In the next step, lidar points were transformed to the image space and the vegetation index of each point is calculated using spectral values and using a certain threshold value, the points that are identified as vegetation and trees are eliminated from the data set. In the final step, by applying the mean shift segmentation algorithm, the set of candidate points of the building are subdivided into separate buildings. As shown in results the proposed method is efficient to the extraction of usual building, although some small buildings with lower heights are not identified and extracted.

\section{REFERENCES}

Akbulut, Z., Özdemir, S., Acar, H., \& Karsli, F. (2018). Automatic Building Extraction from Image and LiDAR Data with Active Contour Segmentation. Journal of the Indian Society of Remote Sensing, 46(12), 2057-2068. https://doi.org/10.1007/s12524-018-0871-2

Awad, M. M. (2017). Toward Robust Segmentation Results Based on Fusion Methods for Very High Resolution Optical Image and LiDAR Data. IEEE Journal of Selected Topics in Applied Earth Observations and Remote Sensing, PP(99), 1-10. https://doi.org/10.1109/JSTARS.2017.2653061

Awrangjeb, M., Fraser, C. S., \& Lu, G. (2015). Building Change Detection From Lidar Point Cloud Data Based on Connected Component Analysis. ISPRS Annals of Photogrammetry, Remote Sensing and Spatial Information Sciences, II-3/W5, 393-400. https://doi.org/10.5194/isprsannals-II-3-W5-393-2015

Awrangjeb, Mohammad, Zhang, C., \& Fraser, C. S. (2013). Automatic extraction of building roofs using LIDAR data and multispectral imagery. ISPRS Journal of Photogrammetry and Remote Sensing, 83, 1-18. https://doi.org/10.1016/j.isprsjprs.2013.05.006

Dal Poz, A. P., \& Fernandes, V. J. M. (2017). BUILDING ROOF BOUNDARY EXTRACTION FROM LIDAR AND IMAGE DATA BASED ON MARKOV RANDOM FIELD. ISPRS - International Archives of the Photogrammetry, Remote Sensing and Spatial Information Sciences, XLII-1/WI(June), 339-344. https://doi.org/10.5194/isprs-archives-XLII-1-W1-3392017

Derpanis, K. G. (2005). Mean Shift Clustering. 1(x), 1-4.

Dong, H. L., Kyoung, M. L., \& Sang, U. L. (2008). Fusion of lidar and imagery for reliable building extraction. Photogrammetric Engineering and Remote Sensing, 74(February), $215-225$ https://doi.org/10.14358/PERS.74.2.215

Feng, M., Zhang, T., Li, S., Jin, G., \& Xia, Y. (2019). An improved minimum bounding rectangle algorithm for regularized building boundary extraction from aerial LiDAR point clouds with partial occlusions.
International Journal of Remote Sensing, 00(00), 1-20. https://doi.org/10.1080/01431161.2019.1641245

Gilani, S. A. N., Awrangjeb, M., \& Lu, G. (2015). Fusion of LiDAR data and multispectral imagery for effective building detection based on graph and connected component analysis. International Archives of the Photogrammetry, Remote Sensing and Spatial Information Sciences - ISPRS Archives, 40(3W2), 65-72. https://doi.org/10.5194/isprsarchives-XL-3-W2-65-2015

Hu, J., You, S., \& Neumann, U. (2000). Approaches to LargeScale Urban Modeling. 62-69.

Huang, J., Zhang, X., Xin, Q., Sun, Y., \& Zhang, P. (2019). Automatic building extraction from high-resolution aerial images and LiDAR data using gated residual refinement network. ISPRS Journal of Photogrammetry and Remote Sensing, 151(September 2018), 91-105. https://doi.org/10.1016/j.isprsjprs.2019.02.019

Huang, R., Yang, B., Liang, F., Dai, W., Li, J., Tian, M., \& Xu, W. (2018). A top-down strategy for buildings extraction from complex urban scenes using airborne LiDAR point clouds. Infrared Physics and Technology, 92(January), 203-218. https://doi.org/10.1016/j.infrared.2018.05.021

Huang, X., Cao, R., \& Cao, Y. (2019). A Density-Based Clustering Method for the Segmentation of Individual Buildings from Filtered Airborne LiDAR Point Clouds. Journal of the Indian Society of Remote Sensing, 47(6), 907-921. https://doi.org/10.1007/s12524-018-0911-y

Liu, J. G., \& Mason, P. J. (2009). Essential Image Processing and GIS for Remote Sensing.

Maltezos, E., \& Ioannidis, C. (2015). Automatic Detection of Building Points From Lidar and Dense Image Matching Point Clouds. ISPRS Annals of Photogrammetry, Remote Sensing and Spatial Information Sciences, II3/W5(October), 33-40. https://doi.org/10.5194/isprsannals-II-3-W5-33-2015

Moussa, A. (2012). A New Object Based Method for Automated Extraction of Urban Objects From Airborne Sensors Data. Isprs Journal of Photogrammetry and Remote Sensing, XXXIX(September), 2008-2013. https://doi.org/10.5194/isprsarchives-XXXIX-B3-3092012

Teo, T. A., \& Chen, L. C. (2004). Object-based building detection from LiDAR data and high resolution satellite imagery. Proceedings of the 25th Asian Conference on Remote Sensing, 1614-1619. Retrieved from http://scholar.google.com/scholar?hl=en\&btnG=Search\& $\mathrm{q}=$ intitle:OBJECT-

BASED+BUILDING+DETECTION+FROM+LIDAR+D ATA+AND+HIGH+RESOLUTION+SATELLITE+IMA GERY\#0

Tomljenovic, I., Tiede, D., \& Blaschke, T. (2016). A building extraction approach for Airborne Laser Scanner data utilizing the Object Based Image Analysis paradigm. International Journal of Applied Earth Observation and Geoinformation, 52, 137-148. https://doi.org/10.1016/j.jag.2016.06.007

Tseng, Y.-H., \& Hung, H.-C. (2016). Extraction of Building Boundary Lines From Airborne Lidar Point Clouds. ISPRS - International Archives of the Photogrammetry, Remote Sensing and Spatial Information Sciences, XLIB3(July), 957-962. https://doi.org/10.5194/isprsarchives-XLI-B3-957-2016

Varghese, V., Shajahan, D. A., \& Nath, A. G. (2017). Building boundary tracing and regularization from LiDAR point cloud. Proceedings of IEEE International Conference on Emerging Technological Trends in Computing, 
Communications and Electrical Engineering, ICETT 2016. https://doi.org/10.1109/ICETT.2016.7873645

Zhang, W., Qi, J., Wan, P., Wang, H., Xie, D., Wang, X., \& Yan, G. (2016). An easy-to-use airborne LiDAR data filtering method based on cloth simulation. Remote Sensing, 8(6), 1-22. https://doi.org/10.3390/rs8060501

Zhou, G., Member, S., \& Zhou, X. (2014). Seamless Fusion of LiDAR and Aerial Imagery for Building Extraction. 115. 